\title{
Oscillation Criteria for Third Order Nonlinear Neutral Differential Equations with Deviating Arguments
}

\author{
E. M. Elabbasy ${ }^{1} \square$ O. Moaaz ${ }^{2}$ \\ Department of Mathematics, Faculty of Science, \\ Mansoura University, Mansoura, 35516, Egypt. \\ 1 emelabbasy@mans.edu.eg; \\ 2 o_moaaz@mans.edu.eg.
}

\begin{abstract}
The aim of this paper is to discuss oscillation and asymptotic behavior of a class of third-order nonlinear neutral delay differential equations. A new theorem is presented that improves a number of results reported in the literature. Example is included to illustrate new results.
\end{abstract}

Keywords: Oscillation, third order, neutral delay differential equations.

\section{Introduction}

In this paper we consider third order neutral differential equations of the form

$$
\begin{aligned}
& \left(r_{2}(t)\left(\left(r_{1}(t)\left(z^{\prime}(t)\right)^{\alpha_{1}}\right)^{\prime}\right)^{\alpha_{2}}\right)^{\prime} \\
& \quad+\int_{a}^{b} q(t, \xi) f(x(g(t, \xi))) d \sigma(\xi)=0, t \geq t_{0},
\end{aligned}
$$

where $z(t)=x(t)+p(t) x(\tau(t))$ and the following conditions are satisfied

$\left(\mathrm{A}_{1}\right)$

$p, \tau \in C(I, \mathrm{R}), o<p(t) \leq p<1, \tau(t) \leq t, \lim _{t \rightarrow \infty} \tau(t)=\infty, \alpha_{1}$

and $\alpha_{2}$ are a quotient of odd positive integers,

$\alpha_{1} \alpha_{2}=\beta$ and $I=\left[t_{0}, \infty\right)$,

$\left(\mathrm{A}_{2}\right) r_{i} \in C(I,(0, \infty)), \int_{t_{0}}^{\infty}\left(r_{i}(t)\right)^{-1 / \alpha_{i}} d t=\infty, i=1,2$,

$\left(\mathrm{A}_{3}\right) f \in C(\mathrm{R}, \mathrm{R}), x f(x)>0$ for $t \geq t_{0}$,

$\left(\mathrm{A}_{4}\right) q \in C(I \times[a, b],[0, \infty)), q(t, \xi)$ is not zero on any half line $\left[t_{\mu}, \infty\right) \times[a, b], t_{\mu} \geq t_{0}$,

$\left(\mathrm{A}_{5}\right) g \in C(I \times[a, b], \mathrm{R}), \quad g(t, \xi) \leq t \quad$ for $t \geq t_{0}$ and $\xi \in[a, b], g(t, \xi)$ is continuous, has positive partial derivative on $I \times[a, b]$ with respect to $t$, nondecreasing with respect to $\xi$ and $\lim _{t \rightarrow \infty} g(t, \xi)=\infty$,

$\left(\mathrm{A}_{6}\right) \sigma \in C([a, b], \mathrm{R}), \sigma$ is nondecreasing and the integral of Eq. (C-1) is in the sense Riemann-stieltijes.
We mean by a solution of Eq. (1.1) a function $x(t):\left[t_{x}, \infty\right) \rightarrow \mathrm{R}, t_{x} \geq t_{0} \quad$ such that $x(t), r_{1}(t)\left(z^{\prime}(t)\right)^{\alpha_{1}}$ and $r_{2}(t)\left(\left(r_{1}(t)\left(z^{\prime}(t)\right)^{\alpha_{1}}\right)^{\prime}\right)^{\alpha_{2}}$ are continuously differentiable for all $t \in\left[t_{x}, \infty\right)$ and $\sup \{|x(t)|: t \geq T\}>0$ for any $T \geq t_{x}$. A solution of Eq. (1.1) is called oscillatory if it has arbitrary large zeros, otherwise it is called nonoscillatory.

Asymptotic properties of solutions of differential equations of the second and third order have been subject of intensive studying in the literature. This problem for neutral differential equations has received considerable attention in recent years (see [1] - [12]).

The aim of this paper is to discuss asymptotic behavior of solutions of class of third order neutral delay differential equation. By using Riccati transformation technique, we established sufficient conditions which insure that solution of class of third order neutral delay differential equation is oscillatory or tended to zero. The results of this study basically generalize and improve the previous results.

Following [Philos [13] ], we introduce a class of functions $\mathfrak{I}$ as follows. Let

$$
D_{0}=\left\{(t, s): t>s>t_{0}\right\} \text { and } D=\left\{(t, s): t \geq s \geq t_{0}\right\}
$$

A kernel function $H \in C(D, \mathbb{R})$ is said to belong to the function class $\mathfrak{I}$, written by $H \in \mathfrak{I}$, if

1) $H(t, s)=0$ for $t \geq t_{0}, H(t, s)>0$ on $D_{0}$

2) $H(t, s)$ has a continuous and nonpositive partial derivative $\partial H / \partial s$ on $D_{0}$ such that the condition 


$$
\frac{\partial H(t, s)}{\partial s}=-h(t, s) H(t, s) \text { for all }(t, s) \in D_{0}
$$

is satisfied for some $h \in C(D, \mathbb{R})$.

Let us state two sets of conditions commonly used, which we rely on:

$$
\begin{aligned}
& \left(\mathrm{S}_{1}\right) \frac{f^{\prime}(x)}{\mid f(x) \frac{1-\beta}{\beta}} \geq k_{1}>0 \text { for } x \neq 0 \text { and } \\
& -f(-u v) \geq f(u v) \geq f(u) f(v) \text { for } u v>0 . \\
& \left(\mathrm{S}_{2}\right) \frac{f(x)}{x^{\beta}} \geq k>0 \text { for } x \neq 0 .
\end{aligned}
$$

For the sake of convenience, we introduce the following notation:

$$
\begin{aligned}
& E_{0}(z(t))=z(t), E_{i}(z(t))=r_{i}(t)\left(\frac{d}{d t} E_{i-1}(z(t))\right)^{\alpha_{i}}, i=1,2, \\
& R\left(t, t_{0}\right)=\left(\frac{1}{r_{1}(t)} \int_{t_{0}}^{t} \frac{1}{r_{2}^{1 / \alpha_{2}}(s)} d s\right)^{1 / \alpha_{1}}, \mu=\frac{\beta^{\beta}}{(\beta+1)^{\beta+1}}
\end{aligned}
$$

and

$$
\widehat{q}(t)=\int_{a}^{b} q(t, \xi) d \sigma(\xi)
$$

and let there exists a function $\rho \in C\left(I, \mathbb{R}^{+}\right)$such that

$$
Q(t, s)=\left|\frac{\rho^{\prime}(s)}{\rho(s)}-h(t, s)\right| .
$$

\section{Several Lemmas}

We begin with some useful lemmas, which we intend to use later.

Lemma 2.1. Assume that $f(y)=U y-V y^{\frac{\eta+1}{\eta}}$, where $U$ and $V$ are constants, $V>0$ and $\eta$ is a quotient of odd positive integers. Then $f$ attends its maximum value on $\mathbb{R}$ at $y^{*}=\left(\frac{U \eta}{V(\eta+1)}\right)^{\eta}$ and

$$
\max _{y \in \mathrm{R}} f=f\left(y^{*}\right)=\frac{\eta^{\eta}}{(\eta+1)^{\eta+1}} U^{\eta+1} V^{-\eta} .
$$

Lemma 2.2. Let $x(t)$ be a positive solution of Eq. (1.1). Then $z(t)$ has only one of the following two properties eventually:

$$
\begin{aligned}
& \left(P_{1}\right) z(t)>0, z^{\prime}(t)>0 \text { and } \frac{d}{d t} E_{1}(z(t))>0, \\
& \left(P_{2}\right) z(t)>0, z^{\prime}(t)<0 \text { and } \frac{d}{d t} E_{1}(z(t))>0 .
\end{aligned}
$$

Proof. Let $x(t)$ be a positive solution of Eq. (1.1). From $\left(A_{1}\right)$ and $\left(A_{5}\right)$, there exists a $t_{1} \geq t_{0}$ such that $x(t)>0, x(\tau(t))>0 \quad$ and $\quad x(g(t, \xi))>0 \quad$ for $t \geq t_{1}$. Then $z(t)>0$ and Eq. (C-1) implies that $\frac{d}{d t} E_{2}(z(t)) \leq 0$. Hence, $E_{2}(z(t))$ is a non-increasing function and of one sign. We claim that $E_{2}(z(t))>0$ for $t \geq t_{1}$. Suppose that $E_{2}(z(t))<0$ for $t \geq t_{2} \geq t_{1}$, then there exists a $t_{3} \geq t_{2}$ and constant $K_{1}>0$ such that

$$
\frac{d}{d t} E_{1}(z(t))<-K_{1}\left(r_{2}(t)\right)^{-1 / \alpha_{2}},
$$

for $t \geq t_{3}$. By integrating the last inequality from $t_{3}$ to $t$, we get

$$
E_{1}(z(t))<E_{1}\left(z\left(t_{3}\right)\right)-K_{1} \int_{t_{3}}^{t}\left(r_{2}(s)\right)^{-1 / \alpha_{2}} d s .
$$

Letting $t \rightarrow \infty$, from $\left(A_{2}\right)$, we have $\lim _{t \rightarrow \infty} E_{1}(z(t))=-\infty$. Then there exists a $t_{4} \geq t_{3}$ and constant $K_{2}>0$ such that

$$
z^{\prime}(t)<-K_{2}\left(r_{1}(t)\right)^{-1 / \alpha_{1}},
$$

for $t \geq t_{4}$. By integrating this inequality from $t_{4}$ to $t$ and using $\left(A_{2}\right)$, we get $\lim _{t \rightarrow \infty} z(t)=-\infty$, which contradicts $z(t)>0$. Now we have $E_{2}(z(t))>0$ for $t \geq t_{1}$. Therefore, $E_{1}(z(t))$ is increasing function. Thus $\left(P_{1}\right)$ or $\left(P_{2}\right)$ holds for $z(t)$, eventually. 


\section{International Journal of Science and Research (IJSR) \\ ISSN (Online): 2319-7064}

Index Copernicus Value (2013): 6.14 $\mid$ Impact Factor (2014): 5.611

Lemma 2.3. Let $\left(S_{1}\right)$ holds, $x(t)$ be a positive solution of Eq. (1.1), and $z(t)$ has the property $\left(P_{2}\right)$. Assume that

$$
\int_{t_{0}}^{\infty}\left(\frac{1}{r_{1}(v)} \int_{v}^{\infty}\left(\frac{1}{r_{2}(u)} \int_{u}^{\infty} \widehat{q}(s) d s\right)^{1 / \alpha_{2}} d u\right)^{1 / \alpha_{1}} d v=\infty
$$

Then the solution $x(t)$ is converges to zero as $t \rightarrow \infty$.

Proof Let $x(t)$ be a positive solution of Eq. (C-1). Since $z(t)$ satisfies the property $\left(P_{2}\right)$, we get

$$
\lim _{t \rightarrow \infty} z(t)=\gamma
$$

Now. We shall prove that $\gamma=0$. Let $\gamma>0$, then we have $\gamma<z(t)<\gamma+\varepsilon$ for all $\varepsilon>0$ and $t$ enough large. Choosing $\varepsilon<\frac{1-p}{p} \gamma$, we obtain

$$
\begin{aligned}
x(t) & =z(t)-p(t) x(\tau(t)) \\
& >\gamma-p z(\tau(t)) \\
& >L(\gamma+\varepsilon)>L z(t),
\end{aligned}
$$

where $L=\frac{\gamma-p(\gamma+\varepsilon)}{\gamma+\varepsilon}>0$. Hence, from (1.1), $\left(S_{1}\right)$ and $\left(A_{5}\right)$, we have

$$
\begin{aligned}
\frac{d}{d t} E_{2}(z(t)) & <-k L^{\beta} \int_{a}^{b} q(t, \xi) z^{\beta}(g(t, \xi)) d \sigma(\xi) \\
& <-k L^{\beta} z^{\beta}(t) \widehat{q}(t) \\
& <-k L^{\beta} \gamma \hat{q}(t) .
\end{aligned}
$$

By integrating two times from $t$ to $\infty$, we get

$$
-z^{\prime}(t)>C\left(\frac{1}{r_{1}(t)} \int_{t}^{\infty}\left(\frac{1}{r_{2}(u)} \int_{u}^{\infty} \widehat{q}(s) d s\right)^{1 / \alpha_{2}} d u\right)^{1 / \alpha_{1}}
$$

where $C=k^{1 / \beta} L \gamma>0$. Integrating the last inequality from $t_{1}$ to $\infty$, we have

$$
z\left(t_{1}\right)>C \int_{t_{1}}^{\infty}\left(\frac{1}{r_{1}(v)} \int_{v}^{\infty}\left(\frac{1}{r_{2}(u)} \int_{u}^{\infty} \hat{q}(s) d s\right)^{1 / \alpha_{2}} d u\right)^{1 / \alpha_{1}} d v
$$

This contradicts to the condition (2.1), then $\lim _{t \rightarrow \infty} z(t)=0$, which implies that $\lim _{t \rightarrow \infty} x(t)=0$.

Lemma 2.4. Let $\left(S_{2}\right)$ holds, $x(t)$ be a positive solution of Eq. (C-1) and $z(t)$ has the property $\left(P_{2}\right)$. If the condition (C-3) holds, then the solution $x(t)$ is converges to zero as $t \rightarrow \infty$.

Proof Proceeding as in the proof of Lemma 2.3. Hence, from (1.1), $\left(S_{2}\right)$ and $\left(A_{5}\right)$, we have

$$
\begin{aligned}
\frac{d}{d t} E_{2}(z(t)) & \leq-\int_{a}^{b} q(t, \xi) f(\operatorname{Lz}(g(t, \xi))) d \sigma(\xi) \\
& <-f(L) f(\gamma) \widehat{q}(t) .
\end{aligned}
$$

The rest of the proof runs as in Lemma 2.4.

\section{Oscillation Theorems}

Theorem 3.1. Let $\left(S_{1}\right)$ and (2.1) hold. If there exist functions $\rho \in C\left(I, \mathbb{R}^{+}\right)$and $H \in \mathfrak{I}$ such that

$$
\limsup _{t \rightarrow \infty} \frac{1}{H\left(t, t_{0}\right)} \int_{t_{0}}^{t} H(t, s)\left(\Theta_{1}(s)-\mu Q^{\beta+1}(t, s) l_{1}(s)\right) d s=\infty
$$

where

$$
\Theta_{1}(t)=\rho(t) \int_{a}^{b} q(t, \xi) f(1-p(g(t, \xi))) d \sigma(\xi),
$$

and

$$
l_{1}(t)=\rho(t)\left(k_{1} R\left(g(t, a), t_{0}\right) g^{\prime}(t, a)\right)^{-\beta} .
$$

Then every solution of Eq. (1.1) is either oscillatory or tends to zero as $t \rightarrow \infty$.

Proof Let $x$ be a non-oscillatory solution of Eq. (1.1) on $I$. Without loss of generality we assume that $x(t) \neq 0$ for $t \geq t_{0}$. Futhermore, we suppose that $x(t)>0$ for $t \geq t_{0}$. Note that $\left(A_{1}\right)$ and $\left(A_{5}\right)$, there exists a $T_{0} \geq t_{0} \quad$ such that $x(t)>0, \quad x(\tau(t))>0$ and $x(g(t, \xi))>0$ for $t \geq T_{0}$. By Lemma C-L2, we have that $z(t)$ has the property $\left(P_{1}\right)$ or the property $\left(P_{2}\right)$.

\section{Volume 4 Issue 12 December 2015}


If $z(t)$ has the property $\left(P_{2}\right)$. Since $(2.1)$ hold, the conditions in Lemma 2.3 are satisfied. Hence, we obtain

$$
\lim _{t \rightarrow \infty} x(t)=0
$$

Now, Let $z(t)$ satisfies the property $\left(P_{1}\right)$, then we have

$$
x(t)=z(t)-p(t) x(\tau(t)) \geq(1-p(t)) z(t)
$$

Thus, from (1.1), $\left(S_{1}\right)$ and $\left(A_{5}\right)$, we have

$\frac{d}{d t} E_{2}(z(t)) \leq-f(z(g(t, a))) \int_{a}^{b} q(t, \xi) f(1-p(g(t, \xi))) d \sigma(\xi)$.

We define

$$
\omega(t)=\rho(t) \frac{E_{2}(z(t))}{f(z(g(t, a)))} .
$$

By differentiating, we get

$$
\begin{aligned}
& \omega^{\prime}(t) \leq \frac{\rho^{\prime}(t)}{\rho(t)} \omega(t)-\Theta_{1}(t) \\
& -\rho(t) \frac{E_{2}(z(t))}{f^{2}(z(g(t, a)))} f^{\prime}(z(g(t, a))) z^{\prime}(g(t, a)) g^{\prime}(t, a) .
\end{aligned}
$$

From $\left(P_{1}\right)$, we have

$$
\begin{aligned}
E_{1}(z(t)) & =E_{1}\left(z\left(t_{1}\right)\right)+\int_{t_{1}}^{t} \frac{E_{2}^{1 / \alpha_{2}}(z(s))}{r_{2}^{1 / \alpha_{2}}(s)} d s \\
& \geq E_{2}^{1 / \alpha_{2}}(z(t)) \int_{t_{1}}^{t} \frac{1}{r_{2}^{1 / \alpha_{2}}(s)} d s,
\end{aligned}
$$

for $t \geq t_{1} \geq T_{0}$. Since $\frac{d}{d t} E_{2}(z(t)) \leq 0$, we obtain

$$
z^{\prime}(g(t, a)) \geq E_{2}^{1 / \beta}(z(t)) R\left(g(t, a), t_{1}\right) .
$$

Hence,

$$
\omega^{\prime}(s) \leq-\Theta_{1}(s)+\frac{\rho^{\prime}(s)}{\rho(s)} \omega(s)-l_{1}^{-1 / \beta}(s) \omega^{\frac{\beta+1}{\beta}}(s),
$$

for $s \geq t_{1}$. Multiplying relation (3.3) by $H(t, s)$ and integrating from $t_{1}$ to $t$, we get

$$
\begin{aligned}
\int_{t_{1}}^{t} H(t, s) \Theta_{1}(s) d s \leq & -\int_{t_{1}}^{t} H(t, s) \omega^{\prime}(s) d s+\int_{t_{1}}^{t} H(t, s) \frac{\rho^{\prime}(s)}{\rho(s)} \omega(s) d s \\
& -\int_{t_{1}}^{t} H(t, s) l_{1}^{-1 / \beta}(s) \omega \omega^{\frac{\beta+1}{\beta}}(s) d s \\
= & H\left(t, t_{1}\right) \omega\left(t_{1}\right)+\int_{t_{1}}^{t} H(t, s)\left(Q(t, s) \omega(s)-l_{1}^{-1 / \beta} \omega^{\frac{\beta+1}{\beta}}\right) d s
\end{aligned}
$$

If $\eta=\beta, U=Q, V=l_{1}^{-1 / \beta}$ and $X=\omega$, then from Lemma 2.1, we obtain

$$
Q \omega-l_{1}^{-1 / \beta} \omega^{\frac{\beta+1}{\beta}} \leq \mu Q^{\beta+1} l_{1} .
$$

Therefore, we get

$$
\omega\left(t_{2}\right) \geq \frac{1}{H\left(t, t_{1}\right)} \int_{t_{1}}^{t} H(t, s)\left(\Theta_{1}(s)-\mu Q^{\beta+1}(t, s) l_{1}(s)\right) d s .
$$

which is contrary to (3.1). This completes the proof of Theorem 3.1.

Theorem 3.2. Let $\left(S_{2}\right)$ and (2.1) hold. If there exist functions $\rho \in C\left(I, \mathbb{R}^{+}\right)$and $H \in \mathfrak{I}$ such that

$$
\limsup _{t \rightarrow \infty} \frac{1}{H\left(t, t_{0}\right)} \int_{t_{0}}^{t} H(t, s)\left(\Theta_{2}(s)-\mu Q^{\beta+1}(t, s) l_{2}(s)\right) d s=\infty,
$$

where

$$
\Theta_{2}(t)=k_{2} \rho(t)(1-p)^{\beta} \widehat{q}(t),
$$

and

$$
l_{1}(t)=\rho(t)\left(\beta R\left(g(t, a), t_{0}\right) g^{\prime}(t, a)\right)^{-\beta} .
$$

Then every solution of Eq. (1.1) is either oscillatory or tends to zero as $t \rightarrow \infty$.

Proof Let $x(t)$ is an eventually positive solution of equation (1.1) on $I$. As in the proof of Theorem C-T1, we have that $z(t)$ has the property $\left(P_{1}\right)$ or the property $\left(P_{2}\right)$. Let $z(t)$ has the property $\left(P_{2}\right)$. From Lemma 3.4, we obtain $\lim _{t \rightarrow \infty} x(t)=0$. On the other hand, when

\section{Volume 4 Issue 12 December 2015}


$z(t)$ satisfies the property $\left(P_{1}\right)$, we have that (3.2) holds. Thus, from (1.1), $\left(S_{1}\right)$ and $\left(A_{5}\right)$, we get

$$
\frac{d}{d t} E_{2}(z(t)) \leq-k_{2}(1-p)^{\beta} z^{\beta}(g(t, a)) \int_{a}^{b} q(t, \xi) d \sigma(\xi) .
$$

We define

$$
\omega(t)=\rho(t) \frac{E_{2}(z(t))}{z^{\beta}(g(t, a))} .
$$

$$
\limsup _{t \rightarrow \infty} \frac{1}{H(t, T)} \int_{T}^{t} \mu H(t, s) Q^{\beta+1}(t, s) l_{1}(s) d s<\infty,
$$

hold. If there exists a function $\psi \in C(I, \mathbb{R})$ such that

$$
\limsup _{t \rightarrow \infty} \int_{T}^{t}\left(\frac{\psi_{+}^{\beta+1}(s)}{l_{1}(s)}\right)^{1 / \beta} d s=\infty
$$

By differentiating, we get

$$
\omega^{\prime}(t) \leq \frac{\rho^{\prime}(t)}{\rho(t)} \omega(t)-\Theta_{2}(t)-\beta \rho(t) \frac{E_{2}(z(t))}{z^{\beta+1}(g(t, a))} z^{\prime}(g(t, a)) g^{\prime}(t, a) .
$$

$\limsup _{t \rightarrow \infty} \frac{1}{H(t, T)} \int_{T}^{t} H(t, s)\left(\Theta_{1}(s)-\mu Q^{\beta+1}(t, s) l_{1}(s)\right) d s \geq \psi(T)$,

Next, by following the same steps in the proof of Theorem 3.1. Hence,

$$
\omega^{\prime}(s) \leq-\Theta_{2}(s)+\frac{\rho^{\prime}(s)}{\rho(s)} \omega(s)-l_{2}^{-1 / \beta}(s) \omega^{\frac{\beta+1}{\beta}}(s),
$$

The rest of the proof runs as in Theorem 3.1. The proof is complete.

Remark If $\quad \alpha_{1}=\alpha_{2}=1, \quad \tau(t)=t-\tau \quad$ and $f(x)=x$. Then, Theorem 3.1 extend Theorem 2.1 in Candan [3].

Remark If $\alpha_{1}=\alpha_{2}=1, a=0, b=1$, $q(t, \xi)=q(t), g(t, \xi)=g(t)$ and $f(x)=x$.Then, Theorem 3.2. extend and improve Theorem 2.1 in Li [9].

Theorem 3.3. Let $\left(S_{1}\right)$ and (2.1) hold. Assume that there exist functions $\rho \in C\left(I, \mathbb{R}^{+}\right)$and $H \in \mathfrak{I}$ such that for every $T \geq t_{0}$,

$$
0<\inf \left[\liminf _{s \geq T} \frac{H(t, s)}{H(t, T)}\right] \leq \infty
$$

and where $\psi_{+}(s)=\max \{\psi(s), 0\}$. Then every solution of Eq. (1.1) is either oscillatory or tends to zero as $t \rightarrow \infty$. Proof As the proof of Theorem 3.1, we can see that (3.3) holds. It follows that

$$
\begin{aligned}
& \limsup _{t \rightarrow \infty} \frac{1}{H\left(t, t_{1}\right)} \int_{t_{1}}^{t} H(t, s)\left(\Theta_{1}(s)-\mu Q^{\beta+1}(t, s) l_{1}(s)\right) d s \\
& \quad \leq \omega\left(t_{1}\right)-\liminf \frac{1}{H\left(t, t_{1}\right)} \int_{t_{1}}^{t} H(t, s)\left(\Phi(t, s)+\mu Q^{\beta+1}(t, s) l_{1}(s)\right) d s,
\end{aligned}
$$

where $\Phi(t, s)=l_{1}^{-1 / \beta}(s) \omega^{\frac{\beta+1}{\beta}}(s)-Q(t, s) \omega(s)$. From (3.8), we have

$$
\begin{gathered}
\liminf _{t \rightarrow \infty} \frac{1}{H\left(t, t_{1}\right)} \int_{t_{1}}^{t} H(t, s)\left(\Phi(t, s)+\mu Q^{\beta+1}(t, s) l_{1}(s)\right) d s \\
\leq \omega\left(t_{1}\right)-\psi\left(t_{1}\right)<\infty .
\end{gathered}
$$

Now, we define functions

$$
F_{1}(t)=\frac{1}{H\left(t, t_{1}\right)} \int_{t_{1}}^{t} H(t, s) Q(t, s) \omega(s) d s
$$

and

$$
F_{2}(t)=\frac{1}{H\left(t, t_{1}\right)} \int_{t_{1}}^{t} H(t, s) l_{1}^{-1 / \beta}(s) \omega^{\frac{\beta+1}{\beta}}(s) d s
$$

From (3.6), we obtain

\section{Volume 4 Issue 12 December 2015}




\section{International Journal of Science and Research (IJSR) \\ ISSN (Online): 2319-7064}

Index Copernicus Value (2013): 6.14 | Impact Factor (2014): 5.611

$$
\liminf \left(F_{2}(t)-F_{1}(t)\right)<\infty
$$

$t \rightarrow \infty$

The remainder of the proof is similar to the theorem 3.1 given in [6] and hence is omitted.

Theorem 3.4. Let $\left(S_{2}\right)$ and (2.1) hold. Assume that there exist functions $\rho \in C\left(I, \mathbb{R}^{+}\right)$and $H \in \mathfrak{I}$ such that for every $T \geq t_{0}, \quad(C-15)$ and

$$
\limsup _{t \rightarrow \infty} \frac{1}{H(t, T)} \int_{T}^{t} \mu H(t, s) Q^{\beta+1}(t, s) l_{2}(s) d s<\infty,
$$

hold. If there exists a function $\psi \in C(I, \mathbb{R})$ such that

$$
\begin{gathered}
\limsup _{t \rightarrow \infty} \int_{T}^{t}\left(\frac{\psi_{+}^{\beta+1}(s)}{l_{2}(s)}\right)^{1 / \beta} d s=\infty, \\
\limsup _{t \rightarrow \infty} \frac{1}{H(t, T)} \int_{T}^{t} H(t, s)\left(\Theta_{2}(s)-\mu Q^{\beta+1}(t, s) l_{2}(s)\right) d s \geq \psi(T),
\end{gathered}
$$

where $\psi_{+}(s)=\max \{\psi(s), 0\}$. Then every solution of Eq. (1.1) is either oscillatory or tends to zero as $t \rightarrow \infty$.

Example Consider the third order neutral delay differential equation

$$
\left(\frac{1}{t}\left(\left(t\left(z^{\prime}(t)\right)^{3}\right)^{\prime}\right)^{1 / 3}\right)^{\prime}+\int_{1 / 2}^{1} \frac{\gamma e^{t(1-\xi)}}{e^{t / 2}-1} x(\xi t) d \xi=0
$$

where $z(t)=x(t)+\frac{1}{2} x\left(\frac{t}{2}\right)$ and $t>0$. Choose $\rho(t)=1, H(t, s)=(t-s)^{2}$ and $k=1$. Hence, we get
It is easy to see that the conditions (2.1) and (3.1) are hold. Then, from Theorem 3.1, every nonoscillatory solution of this equation tends to zero as $t \rightarrow \infty$.

\section{References}

1) B. Baculikova and J. Dzurina, On the asymptotic behavior of a class of third order nonlinear neutral differential equations, Cent. Eur. J. Math. 8(6) -- 2010, 1091-1103.

2) T. Candan, R. S. Dahiya, Functional Differential Equations of Third Order, Electronic Journal of Differential Equations, Conference 12, 2005, pp. 47--56.

3) J. Dzurina, Asymptotic properties of the third delay differential equations, Nonlinear Analysis, 26 (1996), 33--34.

4) E. M. Elabbasy, M.Y. Barsoum and O. Moaaz, Boundedness and Oscillation of Third Order Neutral Differential Equations with Deviating Arguments. Journal of Applied Mathematics and Physics, 3, 13671375 (2015).

5) E. M. Elabbasy, T. S. Hassan, O. Moaaz, Oscillation behavior of second order nonlinear neutral differential equations with deviating arguments, Opuscula Mathematica Vol. 32, No. 4, (2012).

6) E. M. Elabbasy, O. Moaaz, New Oscillation Results for Class of Third Order Neutral Delay Differential Equations with Distributed Deviating Arguments, Global Journal of Science Frontier Research, V. 15, 9, 2015.

7) E. M. Elabbasy, O. Moaaz, On the asymptotic behavior of third-order nonlinear functional differential equations, Serdica Mathematical Journal, (Accepted).

8) E. M. Elabbasy, O. Moaaz, Oscillation theorems for second order neutral differential equations, Jokull Journal (Accepted).

9) T. Li, C. Zhang and G. Xing, Oscillation of Third-Order Neutral Delay Differential Equations, Abstract and Applied Analysis Volume (2012), Article ID 569201, 11 pg.

10) O. Moaaz, Oscillation Theorems for Certain Second Order Differential Equations, Lambert Academic Publishing, Germany (2014).

11) Ch. G. Philos, Oscillation theorems for linear differential equations of second order, Arch. Math. (Basel) 53 (1989) 482--492.

$$
\Theta_{1}(t)=\frac{\gamma}{2 t} \text { and } l_{1}(t)=\frac{4^{4 / 3}}{t}
$$

